\title{
Hertz-Resolution Analog Quadrature Sine Oscillator. Application to Portable Lock-In Amplifiers
}

\author{
Daniel García Romeo, Pedro Martínez, Belén Calvo, Nicolás Medrano \\ Afiliación: Grupo de Diseño Electrónico (GDE) \\ Instituto de Investigación en Ingeniería de Aragón (I3A) \\ Universidad de Zaragoza, Mariano Esquillor s/n, 50018, Zaragoza, Spain. \\ Tel. +34-976762707, e-mail: dgromeo@unizar.es
}

\begin{abstract}
This paper presents the implementation of an analog quadrature sine oscillator capable of generating variable frequency quadrature signals, ranging from 1-100 kHz with less than $2 \mathrm{~Hz}$ resolution and a peak-to-peak voltage of $3 \mathrm{~V}$ operating at a $3.3 \mathrm{~V}$ single supply, to be utilized in high performance portable instrumentation.
\end{abstract}

\section{Motivation}

Lock-in amplifiers (LIA) are a powerful technique to recover information from sensors even though the response of these sensors is too small or is buried in larger noise signals. Although current commercial LIAs are heavy and power-hungry, previous work from the authors proved the implementation of a low-voltage single supply analog LIA processing square wave signals [1-2]., which constitutes an optimal solution for battery-operated microcontrolled systems. However, the use of square signals is not a feasible solution in all sensing applications; it supposes some drawbacks that are needed to be considered, especially when parasite effects are revealed. Thus, a quadrature sine wave generator is needed to develop a general purpose LIA. A review of literature brings out several low-voltage low-power analog structures based on operational amplifiers capable of delivering quadrature signals [3]. The usual frequency control approach involves using variable resistive elements, which implies strong restrictions in terms of magnitude variation and tolerance, rendering an unsuitable solution. Additionally, commercial quadrature oscillators are neither a feasible solution for low-powered system due to its high consumption requirements [4].

It is based on a variable analog oscillator scheme using fast operational amplifiers as basic building blocks, but instead of an analog control method, it exploits a new method to digitally control the quadrature output frequencies that allows reaching the mentioned resolution, overcoming the intrinsic limitations from variable analog oscillators in terms of frequency variation.

\section{Conclusions}

The proposed actuation system, based on an analog oscillator scheme using operational amplifiers as active cells and including a novel digitally controlled frequency approach employing DACs, is shown in Figure 1. It has been implemented in a PCB prototype, using COTS (Figure 2), powered by a $5 \mathrm{~V}$ line from a USB-mini Standard connector. This voltage is reduced to $3.3 \mathrm{~V}$ to feed the rail-torail high slew-rate operational amplifiers MAX4231 from Maxim Integrated. Figure 3 shows the quadrature sine wave outputs VA, VB. These signals present identical amplitude value and a high quality spectrum for a frequency value up to 100 $\mathrm{kHz}$. The spectrum of the output signal, for $80 \mathrm{kHz}$, is plotted in Figure 4; the difference between the first and second harmonic is larger than $40 \mathrm{~dB}$. Figure 5 shows the frequency resolution of the outputs, by using 12 effective bits from the 16-bit DAC. This device can be varied in 28671 positions for a frequency variation of $41610 \mathrm{~Hz}$ achieving a resolution of less than $2 \mathrm{~Hz}$.

\section{REFERENCES}

[1]. AGUIRRE, J. MEDRANO, N. CALVO B. and CELMA, S. A 3V single supply LIA for portable sensing systems. In: Proceedings of IEEE Sensors 2011. Piscataway: IEEE, 2011, pp. 1866-1869.

[2]. AGUIRRE, J. GARCIA-ROMEO, D. MEDRANO, N. CALVO B. and CELMA, S. Square Signal Based Algorithm for Analog Lock-in Amplifiers. IEEE Transactions on Industrial Electronics. 2014, 61(10), 5590-5598.

[3]. SOLIMAN, A.M. Two integrator loop quadrature oscillators: A review. Journal of Advanced Research. 2013, 4(1), 1-11.

[4]. TEXAS INSTRUMENTS. Precision quadrature oscillator. Burr-Brown products from Texas Instruments. Dallas: Texas Instruments, 2004. Operating Manual. 


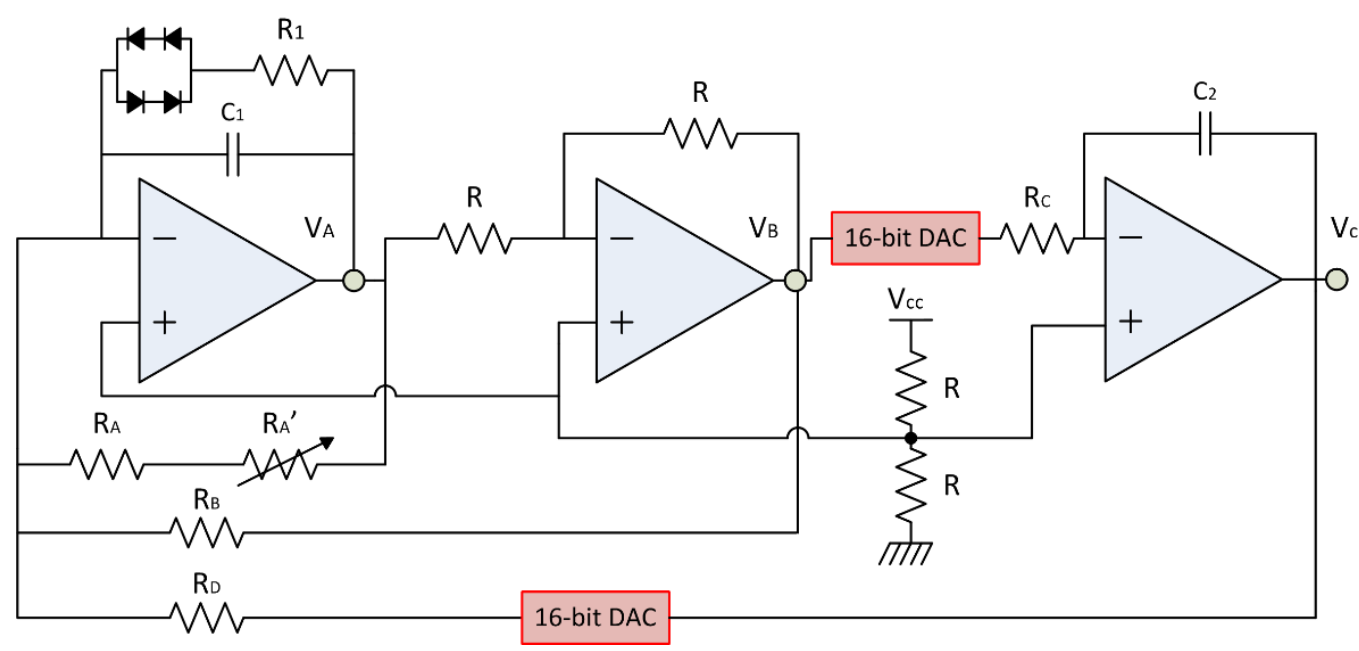

Fig. 1: Quadrature analog sine oscillator structure including a novel digitally controlled frequency approach employing DACs.



Fig. 2: Detailed image of the implemented analog oscillator.



Fig. 3: Sine wave outputs for a frequency value of $80 \mathrm{kHz}$.



Fig. 5: Frequency range reached by the wave outputs compared with the digital words (in decimal representation) programmed in the DAC devices. 